\title{
ON AN ELASTIC DISSIPATION MODEL FOR A CANTILEVERED BEAM
}

\author{
BY \\ W. T. VAN HORSSEN AND M. A. ZARUBINSKAYA
}

Department of Applied Mathematical Analysis, Faculty of Information Technology and Systems, Delft University of Technology, Mekelweg 4, 2628 CD Delft, the Netherlands

\begin{abstract}
In this paper we will study an elastic dissipation model for a cantilevered beam. This problem for a cantilevered beam has been formulated by D. L. Russell as an open problem in $[1,2]$. To determine the relationship between the damping rates and the frequencies we will use a recently developed, adapted form of the method of separation of variables. It will be shown that the dissipation model for the cantilevered beam will not always generate damping. Moreover, it will be shown that some solutions can become unbounded.
\end{abstract}

1. Introduction. It is possible to use different approaches to describe energy dissipation in oscillating, elastic bodies such as beams (see [1,2]). Many approaches (such as molecular theories) are too complicated to use and to analyze in practice. So, as a result, different phenomenological theories are used and applied in mechanics. Of course, every theory has its pros and cons. In particular, Russell notes in $[1,2]$ that it is clear that "viscous" damping models such as

$$
\rho \frac{\partial^{2} u}{\partial t^{2}}+2 \gamma \frac{\partial u}{\partial t}+\frac{\partial^{2}}{\partial x^{2}}\left(E I \frac{\partial^{2} u}{\partial x^{2}}\right)=0
$$

which produce uniform damping rates, are inadequate if experimentally observed damping properties are to be incorporated in the model. Kelvin and Voigt noted at the end of the nineteenth century that damping rates tend to increase with frequency. Incorporated into the Euler-Bernoulli beam model, their approach yields an equation of the form

$$
\rho \frac{\partial^{2} u}{\partial t^{2}}+2 \gamma \rho \frac{\partial^{3}}{\partial t \partial x^{2}}\left(E I \frac{\partial^{2} u}{\partial x^{2}}\right)+\frac{\partial^{2}}{\partial x^{2}}\left(E I \frac{\partial^{2} u}{\partial x^{2}}\right)=0
$$

In [3] Chen and Russell study models of the form

$$
\ddot{x}+B \dot{x}+A x=0,
$$

Received October 30, 2001.

2000 Mathematics Subject Classification. Primary 35B35, 35Q72, 74K10.

E-mail address: W.T.vanHorssen@its.tudelft.nl

E-mail address: maria@dutind4.twi.tudelft.nl 
where $A$ is an elasticity operator and $B$ is related in various ways to the positive square root, $A^{1 / 2}$, of $A$. For beam equations this approach was generalized and developed further by Russell in $[1,2]$. More recent results on the nonnegative square root of fourth order derivative operators are obtained by Yao in [4]. In the last ten years, many other authors (see for instance [7]-[10]) significantly contributed to a deeper understanding of problems which can be formulated by system (1).

In $[1,2]$ Russell introduces a new phenomenological dissipation model for beams, where the damping is assumed to be proportional to the bending rate of the beam. In fact, the following equation is considered:

$$
u_{t t}-\delta u_{t x x}+u_{x x x x}=0,
$$

where $u=u(x, t)$ is the displacement of the beam in vertical direction and $\delta$ is a positive damping constant. No derivation of the dissipation term $u_{t x x}$ is given in $[1,2]$. However, it is noted in $[1,2]$ that this new model has good mathematical properties. For instance, for initial value problems for simply supported beams, such as

$$
\begin{aligned}
& u_{t t}-\delta u_{t x x}+u_{x x x x}=0, \quad 0<x<\pi, \quad t>0, \\
& u(0, t)=u(\pi, t)=u_{x x}(0, t)=u_{x x}(\pi, t)=0, \quad t \geq 0, \\
& u(x, 0)=f(x), \quad u_{t}(x, 0)=g(x), \quad 0<x<\pi,
\end{aligned}
$$

the solution for $0<\delta<2$ is given by

$$
u(x, t)=\sum_{n=1}^{\infty} e^{-\frac{\delta n^{2}}{2} t}\left(A_{n} \sin \left(\frac{n^{2}}{2} \sqrt{4-\delta^{2}} t\right)+B_{n} \cos \left(\frac{n^{2}}{2} \sqrt{4-\delta^{2}} t\right)\right) \sin (n x),
$$

where

$$
A_{n}=\frac{4}{n^{2} \pi \sqrt{4-\delta^{2}}} \int_{0}^{\pi}\left(g(x)+\frac{n^{2}}{2} \delta f(x)\right) \sin (n x) d x, \quad B_{n}=\frac{2}{\pi} \int_{0}^{\pi} f(x) \sin (n x) d x .
$$

Similar formulas can be derived for $\delta \geq 2$.

Indeed it can easily be seen that the damping rate in this case increases with the frequency. Russell [1, 2] and MacCluer [5] observe that the damping operator $B$ in (1) and the stiffness operator $A$ in (1) often "commute", that is, $B$ shares the eigenmodes of $A$, or equivalently the nonnegative square root of the fourth order derivative operator $\frac{\partial^{4} u}{\partial x^{4}}$ is $-\frac{\partial^{2} u}{\partial x^{2}}$. Unfortunately for a cantilevered beam it turns out that $A$ and $B$ do not commute. Russell notes that [2, p. 375]: "The apparent necessity of discarding this model for this reason is a real disappointment ...". For the cantilevered beam Russell shows in [11] that it is not true in general that the energy decreases monotonically, but it remains in [11] an open problem as to how the energy decreases or increases.

In this paper we will study the following initial value problem for a cantilevered beam:

$$
\begin{aligned}
& u_{t t}-\delta u_{t x x}+u_{x x x x}=0, \quad 0<x<\pi, \quad t>0, \\
& u(0, t)=u_{x}(0, t)=u_{x x}(\pi, t)=u_{x x x}(\pi, t)=0, \quad t \geq 0, \\
& u(x, 0)=f(x), \text { and } u_{t}(x, 0)=g(x), \quad 0<x<\pi,
\end{aligned}
$$

where $\delta$ is a positive damping parameter. To find the relationship between the damping rates and the frequencies we will use the recently developed, adapted form of the method of separation of variables (see [6]). This paper is organized as follows. In Sec. 2 of this 
paper we will discuss how this adapted version of the method of separation of variables can be applied to the initial value problem (3) for the cantilevered beam. It will turn out that we have to consider three different cases: $\delta=2, \delta>2$, and $0<\delta<2$. These three cases will be treated in sections 3, 4, and 5 respectively. Finally in Sec. 6 of this paper some conclusions will be drawn and some remarks will be made.

2. On an adapted version of the method of separation of variables. The method of separation of variables is the oldest systematic method to find nontrivial solutions for (linear) partial differential equations. To study waves and vibrations, Daniel Bernoulli, Euler, and D'Alembert used this method in the middle of the eighteenth century. The method has been considerably refined and generalized during the last centuries, and remains a method of great importance and frequent use today. Recently it has been shown in [6] that the method can be applied to a much larger class of problems than is generally assumed. After substitution of a separated solution (that is, a solution of the form $X(x) T(t))$ into the partial differential equation, dividing by $X(x) T(t)$, and after differentiating the so-obtained equation sufficiently many times with respect to some of the independent variables, we can finally reduce the problem to ordinary differential equations. This adapted version of the method of separation of variables seems to be not (well-) known in the literature on partial differential equations. In this section we will show how the adapted method can be applied to the initial value problem (3) for the cantilevered beam.

First we are looking for a nontrivial solution in the form $X(x) T(t)$ which satisfies the partial differential equation (PDE) and the boundary conditions. Substituting this solution form into the PDE, and by dividing the so-obtained equation by $X(x) T(t)$, we find

$$
\frac{\ddot{T}}{T}-\delta \frac{\dot{T}}{T} \frac{X^{\prime \prime}}{X}+\frac{X^{\prime \prime \prime \prime}}{X}=0,
$$

where $^{\prime}=\frac{\partial(\ldots)}{\partial x}$ and ${ }^{\prime}=\frac{\partial(\ldots)}{\partial t}$. Generally it is assumed that (4) cannot be separated because of the mixed term $-\delta \frac{\dot{T}}{T} \frac{X^{\prime \prime}}{X}$. However, by simply differentiating (4) with respect to $x$ or $t$ (see also [6]), we can separate the variables in (4). For instance, if we differentiate (4) with respect to $t$, we obtain

$$
\frac{d}{d t}\left(\frac{\ddot{T}}{T}\right)-\delta \frac{X^{\prime \prime}}{X} \frac{d}{d t}\left(\frac{\dot{T}}{T}\right)=0,
$$

which can easily be separated, yielding

$$
\frac{X^{\prime \prime}}{X}=-\beta
$$

where $\beta$ is a complex valued separation constant. From (5) it follows that $X^{\prime \prime \prime \prime}=$ $-\beta X^{\prime \prime}=\beta^{2} X$, and then it can easily be deduced from (4) that $T(t)$ has to satisfy

$$
\ddot{T}+\delta \beta \dot{T}+\beta^{2} T=0 .
$$

Thus, the problem has been reduced to ordinary differential equations. Finally, by substituting the "separated" solution $X(x) T(t)$ into the boundary conditions, we obtain as 
usual a boundary value problem for $X(x)$ :

$$
\begin{aligned}
& X^{\prime \prime}+\beta X=0, \quad 0<x<\pi, \\
& X(0)=X^{\prime}(0)=X^{\prime \prime}(\pi)=X^{\prime \prime \prime}(\pi)=0,
\end{aligned}
$$

where $\beta$ is a complex valued separation constant. It turns out that the boundary value problem (7) only has trivial solutions. We will omit these lengthy but elementary calculations. So, differentiation of (4) with respect to $t$ leads for the cantilevered beams to trivial solutions. For a simply supported beam, however, it will lead to the following boundary value problem for $X(x)$ :

$$
\begin{aligned}
& X^{\prime \prime}+\beta X=0, \quad 0<x<\pi, \\
& X(0)=X^{\prime \prime}(0)=X(\pi)=X^{\prime \prime}(\pi)=0,
\end{aligned}
$$

which has nontrivial solutions $X(x)=\sin (n x)$ for $n=1,2,3 \ldots$ These solutions will finally lead to the solution of the initial value problem for the simply supported beam as, for instance, given by (2) for $0<\delta<2$.

We can also differentiate (4) with respect to $x$ to obtain

$$
-\delta \frac{\dot{T}}{T} \frac{d}{d x}\left(\frac{X^{\prime \prime}}{X}\right)+\frac{d}{d x}\left(\frac{X^{\prime \prime \prime \prime}}{X}\right)=0,
$$

which can also easily be separated, yielding

$$
\frac{\dot{T}}{T}=\lambda,
$$

where $\lambda$ is a complex valued separation constant. From (8) it follows that $\ddot{T}=\lambda \dot{T}=\lambda^{2} T$, and then it can easily be deduced from $(4)$ that $X(x)$ has to satisfy the following boundary value problem:

$$
\begin{aligned}
& X^{\prime \prime \prime \prime}-\delta \lambda X^{\prime \prime}+\lambda^{2} X=0, \quad 0<x<\pi, \\
& X(0)=X^{\prime}(0)=X^{\prime \prime}(\pi)=X^{\prime \prime \prime}(\pi)=0,
\end{aligned}
$$

where $\lambda=\lambda_{1}+i \lambda_{2}$ with $\lambda_{1}$ and $\lambda_{2} \in \mathbb{R}$. By considering the characteristic equation

$$
k^{4}-\delta \lambda k^{2}+\lambda^{2}=0 \Longleftrightarrow\left(k^{2}-\frac{\delta \lambda}{2}\right)^{2}+\frac{\lambda^{2}}{4}\left(4-\delta^{2}\right)=0
$$

for the differential equation in (9), it is obvious that we have to consider three cases: $\delta=2, \delta>2$, and $0<\delta<2$. These three cases will be studied in the next three sections. It will be shown that nontrivial solutions for (9) can be found in all three cases. From (8) the time-dependent behaviour of a nontrivial solution $X(x) T(t)$ for (3) can be determined. It is obvious from (8) that arbitrary vibrations of the cantilevered beam can only be damped out if all eigenvalues $\lambda$ have a negative real part, that is, $\lambda_{1}$ should be negative for all vibration modes.

3. The case $\delta=2$. In this section we will study the boundary value problem (9) with $\delta=2$. The characteristic equation for the differential equation in (9) becomes in this case

$$
\left(k^{2}-\lambda\right)^{2}=0
$$


where $\lambda=\lambda_{1}+i \lambda_{2}$ with $\lambda_{1}$ and $\lambda_{2} \in \mathbb{R}$. It can be shown elementarily that for $\lambda_{2}=0$, the boundary value problem (9) has only trivial solutions. For $\lambda_{2} \neq 0$, the characteristic equation (11) has as roots

$$
\zeta_{1}+i \zeta_{2}, \quad \text { and } \quad-\zeta_{1}-i \zeta_{2}
$$

where

$$
\zeta_{1}=\sqrt{\frac{\sqrt{\lambda_{1}^{2}+\lambda_{2}^{2}}+\lambda_{1}}{2}}, \zeta_{2}=\sqrt{\frac{\sqrt{\lambda_{1}^{2}+\lambda_{2}^{2}}-\lambda_{1}}{2}} .
$$

Each root has multiplicity two. Putting $k_{1}=\zeta_{1}+i \zeta_{2}$, the solution of the differential equation in (9) can now be written as

$$
X(x)=C_{1} \cosh \left(k_{1} x\right)+C_{2} \sinh \left(k_{1} x\right)+C_{3} x \cosh \left(k_{1} x\right)+C_{4} x \sinh \left(k_{1} x\right),
$$

where $C_{1}, C_{2}, C_{3}$, and $C_{4}$ are complex valued constants of integration. By substituting (13) into the boundary conditions in (9) we obtain a system of four linear, homogeneous equations for $C_{1}, C_{2}, C_{3}$, and $C_{4}$. To have a nontrivial solution the determinant of the coefficient matrix has to be zero, yielding

$$
\sinh ^{2}\left(k_{1} \pi\right)-k_{1}^{2} \pi^{2}+4=0 .
$$

Taking apart real and imaginary parts in (14) we get a system of two nonlinear equations for $\zeta_{1}$ and $\zeta_{2}$ (note that $\left.k_{1}=\zeta_{1}+i \zeta_{2}\right)$ :

$$
\begin{aligned}
& \cosh \left(2 \pi \zeta_{1}\right) \cos \left(2 \pi \zeta_{2}\right)=\frac{1}{2}\left(\left(2 \pi \zeta_{1}\right)^{2}-\left(2 \pi \zeta_{2}\right)^{2}\right)-7, \\
& \sinh \left(2 \pi \zeta_{1}\right) \sin \left(2 \pi \zeta_{2}\right)=2 \pi \zeta_{1} 2 \pi \zeta_{2} .
\end{aligned}
$$

Using the formula manipulation package Maple, numerical approximations of the solution of (15) can easily be obtained. Using these approximations and (12), the eigenvalues $\lambda=\lambda_{1}+i \lambda_{2}$ can be approximated. The first six approximations of the eigenvalues $\lambda$ of the boundary value problem (9) are listed in Table 1.

\begin{tabular}{|c|r|r|}
\hline & \multicolumn{2}{|c|}{$\delta=2$} \\
\hline Nr. & \multicolumn{1}{|c|}{$\lambda_{1}$} & \multicolumn{1}{c|}{$\lambda_{2}$} \\
\hline 1 & 0.072471 & 0.327553 \\
2 & -1.306096 & 2.005633 \\
3 & -4.930357 & 4.225684 \\
4 & -10.658699 & 6.772424 \\
5 & -18.442296 & 9.470416 \\
6 & -28.260886 & 12.309142 \\
\hline
\end{tabular}

TABLE 1. Approximations of the first six eigenvalues $\lambda=\lambda_{1}+i \lambda_{2}$ for the case $\delta=2$.

The first eigenvalue has a positive real part. From (8) it can readily be seen that for this eigenvalue there exists a nontrivial solution $X(x) T(t)$ of $(3)$ which becomes unbounded for increasing times $t$. So for this first vibration mode there certainly is no energy dissipation. 
4. The case $\delta>2$. In this section we will study the boundary value problem (9) with $\delta>2$. The characteristic equation for the differential equation in (9) is

$$
k^{4}-\lambda \delta k^{2}+\lambda^{2}=0,
$$

where $\lambda=\lambda_{1}+i \lambda_{2}$ with $\lambda_{1}$ and $\lambda_{2} \in \mathbb{R}$. It is easy to show that for $\lambda_{2}=0$ the boundary value problem (9) has only trivial solutions. For $\lambda_{2} \neq 0$ it follows from the characteristic equation (16) that

$$
k^{2}=\lambda\left(\frac{\delta}{2}+\frac{1}{2} \sqrt{\delta^{2}-4}\right), \quad \text { or } \quad k^{2}=\frac{\lambda}{\left(\frac{\delta}{2}+\frac{1}{2} \sqrt{\delta^{2}-4}\right)} .
$$

Putting $a=\left(\frac{\delta}{2}+\frac{1}{2} \sqrt{\delta^{2}-4}\right)$ it follows from (17) that $k^{2}=\lambda a$ or $k^{2}=\frac{\lambda}{a}$. And so, the roots of the characteristic equation (16) are

$$
a p_{1},-a p_{1}, p_{1} \text {, and } p_{1},
$$

where $p_{1}=\xi_{1}+i \xi_{2}$ with

$$
\xi_{1}=\sqrt{\frac{\sqrt{\lambda_{1}^{2}+\lambda_{2}^{2}}+\lambda_{1}}{2}} \text { and } \xi_{2}=\sqrt{\frac{\sqrt{\lambda_{1}^{2}+\lambda_{2}^{2}}-\lambda_{1}}{2}} .
$$

For $\lambda_{2} \neq 0$ the solution of the differential equation in (9) can now be written as

$$
X(x)=C_{1} \cosh \left(p_{1} x\right)+C_{2} \sinh \left(p_{1} x\right)+C_{3} \cosh \left(a p_{1} x\right)+C_{4} \sinh \left(a p_{1} x\right),
$$

where $C_{1}, C_{2}, C_{3}$, and $C_{4}$ are complex valued constants of integration. By substituting (19) into the boundary conditions in (9) we obtain a system of four linear, homogeneous equations for $C_{1}, C_{2}, C_{3}$, and $C_{4}$. To have a nontrivial solution the determinant of the coefficient matrix has to be zero, yielding

$$
1+a^{4}+\frac{a}{2}(a-1)^{2} \cosh \left((a+1) p_{1} \pi\right)-\frac{a}{2}(a+1)^{2} \cosh \left((a-1) p_{1} \pi\right)=0 .
$$

Taking apart the real and imaginary parts in (20) we finally obtain a system of two nonlinear equations for $\xi_{1}$ and $\xi_{2}$ (note that $p_{1}=\xi_{1}+i \xi_{2}$ )

$$
\begin{aligned}
& 1+a^{4}+\frac{a}{2}(a-1)^{2} \cosh \left((a+1) \pi \xi_{1}\right) \cos \left((a+1) \pi \xi_{2}\right)-\frac{a}{2}(a+1)^{2} \cosh \left((a-1) \pi \xi_{1}\right) \cos \left((a-1) \pi \xi_{2}\right)=0, \\
& \frac{a}{2}(a-1)^{2} \sinh \left((a+1) \pi \xi_{1}\right) \sin \left((a+1) \pi \xi_{2}\right)-\frac{a}{2}(a+1)^{2} \sinh \left((a-1) \pi \xi_{1}\right) \sin \left((a-1) \pi \xi_{2}\right)=0
\end{aligned}
$$

Numerical approximations of the solution of (21) can easily be obtained by using the formula manipulation package Maple. Using these approximations and (18) we can approximate the eigenvalues $\lambda=\lambda_{1}+i \lambda_{2}$. The first six approximations of the eigenvalues $\lambda$ are listed in Table 2 for some specific values of the parameter $\delta>2$.

From Table 2 it follows that for each specific value $\delta>2$ (as listed in Table 2), the first eigenvalue always has a positive real part. From (8) it can readily be seen that for these first eigenvalues there exist nontrivial solutions $X(x) T(t)$ of $(3)$ which become unbounded for increasing times $t$. So for this first vibration mode there certainly is no energy dissipation. 


\begin{tabular}{|c|r|r|r|r|r|c|}
\hline & \multicolumn{2}{|c|}{$\delta=2.001$} & \multicolumn{2}{c|}{$\delta=3$} & \multicolumn{2}{c|}{$\delta=10$} \\
\hline Nr. & \multicolumn{1}{|c|}{$\lambda_{1}$} & \multicolumn{1}{c|}{$\lambda_{2}$} & \multicolumn{1}{c|}{$\lambda_{1}$} & $\lambda_{2}$ & $\lambda_{1}$ & $\lambda_{2}$ \\
\hline 1 & 0.071093 & 0.318035 & 0.035470 & 0.058214 & 0.012281 & 0.019740 \\
2 & -1.266093 & 1.943048 & -0.675846 & 0.582716 & -0.054805 & 0.055348 \\
3 & -4.779897 & 4.122121 & -1.728249 & 0.623632 & -0.197794 & 0.087744 \\
4 & -10.334167 & 6.553759 & -3.707961 & 1.393278 & -0.418701 & 0.130915 \\
5 & -18.384359 & 9.277380 & -6.116124 & 1.063614 & -0.705594 & 0.202380 \\
6 & -27.403149 & 11.889795 & -9.027316 & 2.169593 & -0.994408 & 0.285117 \\
\hline
\end{tabular}

TABLE 2. Approximations of the first six eigenvalues $\lambda=\lambda_{1}+i \lambda_{2}$ for some specific values of the parameter $\delta>2$.

5. The case $0<\delta<2$. In this section we will study the boundary value problem (9) with $0<\delta<2$. The characteristic equation for the differential equation in (9) has the form (16). It can be shown elementarily that for $\lambda_{2}=0$ the boundary value problem (9) has only trivial solutions. For $\lambda_{2} \neq 0$ it follows from the characteristic equation (16) that

$$
k^{2}=\lambda\left(\frac{\delta}{2}+i \frac{\sqrt{4-\delta^{2}}}{2}\right), \quad \text { or } \quad k^{2}=\frac{\lambda}{\frac{\delta}{2}+i \frac{\sqrt{4-\delta^{2}}}{2}}
$$

Putting $a=\frac{\delta}{2}+i \frac{\sqrt{4-\delta^{2}}}{2}$, it follows from (22) that $k^{2}=\lambda a$, or $k^{2}=\frac{\lambda}{a}$. And so, the roots of the characteristic equation (16) are

$$
a p_{1},-a p_{1}, p_{1},-p_{1},
$$

where $p_{1}=\eta_{1}+i \eta_{2}$ with

$$
\begin{aligned}
& \eta_{1}=\frac{1}{2}\left(\sqrt{\frac{\sqrt{\lambda_{1}^{2}+\lambda_{2}^{2}}+\lambda_{1}}{2}(2+\delta)}-\sqrt{\frac{\sqrt{\lambda_{1}^{2}+\lambda_{2}^{2}}-\lambda_{1}}{2}(2-\delta)}\right), \quad \text { and } \\
& \eta_{2}=\frac{1}{2}\left(\sqrt{\frac{\sqrt{\lambda_{1}^{2}+\lambda_{2}^{2}}-\lambda_{1}}{2}(2+\delta)}+\sqrt{\frac{\sqrt{\lambda_{1}^{2}+\lambda_{2}^{2}}+\lambda_{1}}{2}(2-\delta)}\right) .
\end{aligned}
$$

As in Sec. 4, the solution of the differential equation in (9) can be written in the form (19). Again we obtain a system of four linear, homogeneous equations for $C_{1}, C_{2}, C_{3}$, and $C_{4}$ by substituting (19) into the boundary conditions in (9). To have a nontrivial solution the determinant of the coefficient matrix has to be zero, yielding (20). The only difference now with the previous section is that $a$ and $p_{1}$ are both complex valued. Taking apart the real and imaginary parts in Eq. (20) we obtain a system of two nonlinear equations 
for $\eta_{1}$ and $\eta_{2}$ (note that $\left.p_{1}=\eta_{1}+i \eta_{2}\right)$ :

$$
\begin{aligned}
& \left(\delta^{2}-2\right)^{2} \\
& +\left(\delta^{2} / 2-1\right)(\delta-2) \cosh \left(\left((\delta / 2+1) \eta_{1}-\eta_{2} \sqrt{4-\delta^{2}} / 2\right) \pi\right) \cos \left(\left((\delta / 2+1) \eta_{2}+\eta_{1} \sqrt{4-\delta^{2}} / 2\right) \pi\right) \\
& -\delta \sqrt{4-\delta^{2}}(\delta-2) / 2 \sinh \left(\left((\delta / 2+1) \eta_{1}-\eta_{2} \sqrt{4-\delta^{2}} / 2\right) \pi\right) \sin \left(\left((\delta / 2+1) \eta_{2}+\eta_{1} \sqrt{4-\delta^{2}} / 2\right) \pi\right) \\
& -\left(\delta^{2} / 2-1\right)(\delta+2) \cosh \left(\left((\delta / 2-1) \eta_{1}-\eta_{2} \sqrt{4-\delta^{2}} / 2\right) \pi\right) \cos \left(\left((\delta / 2-1) \eta_{2}+\eta_{1} \sqrt{4-\delta^{2}} / 2\right) \pi\right) \\
& +\delta \sqrt{4-\delta^{2}}(\delta+2) / 2 \sinh \left(\left((\delta / 2-1) \eta_{1}-\eta_{2} \sqrt{4-\delta^{2}} / 2\right) \pi\right) \sin \left(\left((\delta / 2-1) \eta_{2}+\eta_{1} \sqrt{4-\delta^{2}} / 2\right) \pi\right)=0, \\
& \text { and } \\
& \delta \sqrt{4-\delta^{2}}\left(\delta^{2}-2\right) \\
& +\delta \sqrt{4-\delta^{2}}(\delta-2) / 2 \cosh \left(\left((\delta / 2+1) \eta_{1}-\eta_{2} \sqrt{4-\delta^{2}} / 2\right) \pi\right) \cos \left(\left((\delta / 2+1) \eta_{2}+\eta_{1} \sqrt{4-\delta^{2}} / 2\right) \pi\right) \\
& -\left(\delta^{2} / 2-1\right)(\delta-2) \sinh \left(\left((\delta / 2+1) \eta_{1}-\eta_{2} \sqrt{4-\delta^{2}} / 2\right) \pi\right) \sin \left(\left((\delta / 2+1) \eta_{2}+\eta_{1} \sqrt{4-\delta^{2}} / 2\right) \pi\right) \\
& -\delta{\sqrt{4-\delta^{2}}}^{2}(\delta+2) / 2 \cosh \left(\left((\delta / 2-1) \eta_{1}-\eta_{2} \sqrt{4-\delta^{2}} / 2\right) \pi\right) \cos \left(\left((\delta / 2-1) \eta_{2}+\eta_{1} \sqrt{4-\delta^{2}} / 2\right) \pi\right) \\
& -\left(\delta^{2} / 2-1\right)(\delta+2) \sinh \left(\left((\delta / 2-1) \eta_{1}-\eta_{2} \sqrt{4-\delta^{2}} / 2\right) \pi\right) \sin \left(\left((\delta / 2-1) \eta_{2}+\eta_{1} \sqrt{4-\delta^{2}} / 2\right) \pi\right)=0 \text {. }
\end{aligned}
$$

Using the formula manipulation package Maple, numerical approximations of the solution of (24) can easily be obtained. Using these approximations and (23), the eigenvalues $\lambda=\lambda_{1}+i \lambda_{2}$ can be approximated. The first six approximations of the eigenvalues $\lambda$ of the boundary value problem (9) are listed in Table 3 for some specific values of the parameter $0<\delta<2$.

\begin{tabular}{|c|r|r|r|r|r|r|}
\hline & \multicolumn{2}{|c|}{$\delta=0.01$} & \multicolumn{2}{c|}{$\delta=1$} & \multicolumn{1}{c|}{$\delta=1.999$} \\
\hline Nr. & \multicolumn{1}{|c|}{$\lambda_{1}$} & \multicolumn{1}{c|}{$\lambda_{2}$} & \multicolumn{1}{c|}{$\lambda_{1}$} & \multicolumn{1}{c|}{$\lambda_{2}$} & \multicolumn{1}{c|}{$\lambda_{1}$} & \multicolumn{1}{c|}{$\lambda_{2}$} \\
\hline 1 & 0.000438 & 0.356246 & 0.041226 & 0.347651 & 0.072445 & 0.327575 \\
2 & -0.006735 & 2.232556 & -0.669480 & 2.183093 & -1.305498 & 2.005905 \\
3 & -0.023255 & 6.251193 & -2.352370 & 5.821765 & -4.927529 & 4.259369 \\
4 & -0.050113 & 12.249825 & -5.049603 & 11.194332 & -10.651827 & 6.780897 \\
5 & -0.086926 & 20.249820 & -13.444049 & 27.132464 & -18.429408 & 9.490139 \\
6 & -0.133743 & 30.249707 & -19.141120 & 37.699619 & -28.239893 & 12.347001 \\
\hline
\end{tabular}

TABLE 3. Approximations of the first six eigenvalues $\lambda=\lambda_{1}+i \lambda_{2}$ for some specific values of the parameter $0<\delta<2$.

From Table 3 it follows that for each specific value $0<\delta<2$ (as listed in Table 3 ), the first eigenvalue always has a positive real part. From (8) it can readily be seen that for these first eigenvalues there exist nontrivial solutions $X(x) T(t)$ of $(3)$ which become unbounded for increasing times $t$. So for this first vibration mode there certainly is no energy dissipation.

6. Conclusion. In this paper a phenomenological dissipation model for a cantilevered beam has been studied. This new model has been introduced in $[1,2]$ as a model for a beam where the damping is assumed to be proportional to the bending rate of the beam. For the cantilevered beam the relationship between the damping rates and the frequencies has been obtained by using the recently developed, adapted version of the method 
of separation of variables (see [6]). It should be remarked that this relationship also can be obtained by applying the Laplace transformation method to (3). The boundary value problem (9) then also is obtained.

It has been shown that this phenomenological model for the cantilevered beam does not always generate damping. For some significant values of the "damping" parameter $\delta$ is has been shown numerically that the first (that is, the lowest) vibration mode is unstable. So for this mode there certainly is no energy dissipation. From this point of view we have to conclude that the elastic dissipation model for the cantilevered beam is not an adequate dissipation model.

\section{REFERENCES}

[1] D. L. Russell, On the positive root of the fourth derivative operator, Quarterly of Applied Mathematics, vol. XVI, No. 4, (1988), p. 751-773.

[2] D. L. Russell, A comparison of certain elastic dissipation mechanisms via decoupling and projection techniques, Quarterly of Applied Mathematics, Vol. XIX, No. 2, (1991), p. 373-396.

[3] G. Chen and D. L. Russell, A mathematical model for linear elastic systems with structural damping, Quarterly of Applied Mathematics, Vol. XVI, No. 1, (1982), p. 433-454.

[4] P. F. Yao, On the negative square root of the fourth derivative operators, J. of Differential Equations 148, (1998), p. 318-333.

[5] C. R. MacCluer, Boundary value problems and orthogonal expansions, New York, IEEE Press, (1994).

[6] W. T. van Horssen, On the applicability of the method of separation of variables for partial difference equations, Journal of Difference Equations and Applications, vol. 8, No. 1, (2002), p. 53-60.

[7] S. Chen, K. Liu, and Z. Liu, Spectrum and stability for elastic systems with global or local KelvinVoigt damping, SIAM J. on Applied Mathematics, vol. 59, No. 2, (1998), p. 651-668.

[8] S. Chen and R. Triggiani, Proof of extensions of two conjectures on structural damping for elastic systems, Pacific Journal of Mathematics, vol. 136, No. 1, (1989), p. 15-55.

[9] S. Chen and R. Triggiani, Characterization of domains of fractional powers of certain operators arising in elastic systems, and applications, J. of Differential Equations, 88 (1990), p. 279-293.

[10] S. Chen and R. Triggiani, Gevrey class semigroups arising from elastic systems with gentle dissipation: the case $0<\alpha<\frac{1}{2}$, Proceedings of the American Mathematical Society, Vol. 110, No. 2 (1990), p. 401-415.

[11] D. L. Russell, On mathematical models for the elastic beam with frequency-proportional damping, Chapter 4 in Control and Estimation in Disturbed Parameter Systems, edited by H. T. Banks, vol. 11 of Frontiers in Applied Mathematics, SIAM Pubs., 1992. 